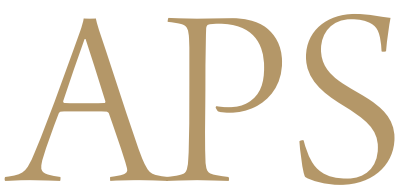

Archives of Plastic Surgery

\title{
Immediate Debridement and Reconstruction with a Pectoralis Major Muscle Flap for Poststernotomy Mediastinitis
}

\author{
Yu Jin Jang, Myong Chul Park, Dong Ha Park, Hyoseob Lim, Joo Hyoung Kim, Il Jae Lee \\ Department of Plastic and Reconstructive Surgery, Ajou University Hospital, Ajou University School of Medicine, Suwon, Korea
}

Background Poststernotomy mediastinitis is a rare, but life-threatening complication, thus early diagnosis and proper management is essential for poststernotomy mediastinitis. The main treatment for mediastinitis is aggressive debridement. Several options exist for reconstruction of defects after debridement. The efficacy of immediate debridement and reconstruction with a pectoralis major muscle flap designed for the defect immediately after the diagnosis of poststernotomy mediastinitis is demonstrated.

Methods Between September 2009 and June 2011, 6 patients were referred to the Department of Plastic and Reconstructive Surgery and the Department of Thoracic and Cardiovascular Surgery of Ajou University Hospital for poststernotomy mediastinitis. All of the patients underwent extensive debridement and reconstruction with pectoralis major muscle flaps, advanced based on the pedicle of the thoracoacromial artery as soon as possible following diagnosis. A retrospective review of the 6 cases was performed to evaluate infection control, postoperative morbidity, and mortality.

Results All patients had complete wound closures and reduced severity of infections based on the erythrocyte sedimentation rate and C-reactive protein levels and a reduction in poststernal fluid collection on computed tomography an average of 6 days postoperatively. A lack of growth of organisms in the wound culture was demonstrated after 3 weeks. There were no major wound morbidities, such as hematomas, but one minor complication required a skin graft caused by skin flap necrosis. No patient expired after definitive surgery.

Conclusions Immediate debridement and reconstruction using a pectoralis major muscle flap is a safe technique for managing infections associated with poststernotomy mediastinitis, and is associated with minimal morbidity and mortality.

Keywords Mediastinitis / Pectoralis muscle / Pedicled flap
Correspondence: II Jae Lee Department of Plastic and Reconstructive Surgery, Ajou University Hospital, Ajou University School of Medicine, 164 Worldcup-ro, Yeongtong-gu, Suwon 443-721, Korea Tel: +82-31-219-5614

Fax: +82-31-219-5610

E-mail: i00325@live.co.kr

Received: 7 Sep 2011 • Revised: 24 Dec 2011 • Accepted: 28 Dec 2011

pISSN: 2234-6163・ elSSN: 2234-6171 • http://dx.doi.org/10.5999/aps.2012.39.1.36• Arch Plast Surg 2012;39:36-41

\section{INTRODUCTION}

Poststernotomy mediastinitis is a life-threatening complication after cardiac surgery. The incidence of poststernotomy mediastinitis is low, ranging between $0.25 \%$ and $8 \%$, but resulting in a high mortality rate (5\% to $50 \%)$ [1]. The definition of poststernotomy mediastinitis has been established by the US Centers for Disease Control and Prevention [2]. According to the guidelines, the diagnosis of mediastinitis requires at least one of the following: 1) an organism isolated from cultures of mediastinal 
tissue or fluids; 2) evidence of mediastinitis intra-operatively; 3 ) and chest pain, sternal instability, or fever $\left(>38^{\circ} \mathrm{C}\right)$, in combination with purulent discharge from the mediastinum or an organism isolated from the blood cultures or cultures of mediastinal drainage.

The initial approach for the primary treatment of mediastinitis is rigorous debridement of the necrotic tissues, including the bone. The bacterial innoculum is reduced locally by the debridement of all infected and necrotic soft tissues. A critical bacterial count of $10^{5}$ microorganisms per gram of tissue has been associated with the development of infection, thus it has been reported that successful control of infection occurred if the counts were $<10^{5}$ microorganisms per gram of tissue [3]. Various methods of reconstruction of the mediastinal defect after debridement of poststernotomy mediastinitis have been described over the past 25 years, including muscle flaps, omental flaps, and vacuum-assisted closure $[4,5]$. The pectoralis major muscle flap is considered the main method of reconstruction. In this study, the results of deep sternal wound infections with poststernotomy mediastinitis, which were reconstructed with pectoralis major flaps designed for the defects after debridement immediately at the time the diagnosis made, were evaluated.

\section{METHODS}

A retrospective review was made of 6 patients referred to the Department of Plastic and Reconstructive Surgery for mediastinitis after sternotomies performed in the Department of Thoracic and Cardiovascular Surgery between December 2009 and June 2011. Patient age, gender, diagnosis, surgical method, the time of presentation of the deep sternal wound infection, and the time of referral to the Department of Plastic and Reconstructive Surgery were recorded. Once the diagnosis of poststernotomy mediastinitis was confirmed, each of the patients was immediately managed surgically by the plastic surgeon. The extent of infection was assessed based on inflammatory markers (erythrocyte sedimentation rate $[\mathrm{ESR}]$ and C-reactive protein $[\mathrm{CRP}])$, fever, organism isolated from a culture of the mediastinal drainage, and imaging (computed tomography, CT). In addition, sternal instability was evaluated based on the CT. Wound cultures were taken from the sternum within 3 days before and every week after surgery; the cultures included intraoperative deep tissue culture, which then could be used to assess the sensitivity of postoperative antibiotic therapy. The most relevant information about the patients is summarized in Table 1.

\section{Surgical procedures}

General anesthesia was administered to all 6 patients prior to surgery. The incision was centered over the skin fistula and extended to the sternum. Necrotic skin and hyperplastic tissue of the fistula were debrided. All wires were removed and wound cultures were taken. After complete debridement until healthy solid bone with bleeding margins was found, the size and shape of the defect was measured. The pectoralis major muscle was dissected from the overlying skin and released from the sternal insertions without cutting the humeral attachment, so as to preserve sufficient mobility of the arm, and rotated based on the pedicle of the thoracoacromial artery (Figs. 1, 2). Sternal fixation was performed with wires when bone stability was uncertain. A closed suction drain was placed in the mediastinum and two additional drains were inserted laterally, with one under each flap. The flap was designed to fit the defect of the wound, advanced medially without tension for obliteration of the cavity of the sternal wound, and packed into the dead space if needed (Fig. 3). Their overlying fascia and skin were then closed in layers over the closed suction drains. Antibiotics were administered according to the microbiologic results of wound culture. Adduction of both upper arms was maintained for 2 weeks postoperatively.

\section{RESULTS}

There were four men and two women enrolled in the study, rang-

\section{Table 1. Patient data}

\begin{tabular}{|c|c|c|c|c|c|c|}
\hline Case & $\begin{array}{l}\text { Age } \\
\text { (yr) }\end{array}$ & Sex & Diagnosis & Previous surgery & $\begin{array}{c}\text { Mediastinitis } \\
\text { after sternotomy } \\
\text { (day) }\end{array}$ & $\begin{array}{c}\text { Debridement } \\
\text { reconstruction } \\
\text { after diagnosis (day) }\end{array}$ \\
\hline 1 & 55 & $\mathrm{M}$ & AMI & OPCAB & 7 & 0 \\
\hline 2 & 65 & M & AMI & ОРСАВ & 9 & 2 \\
\hline 3 & 68 & $\mathrm{M}$ & AMI & ОРСАВ & 37 & 1 \\
\hline 4 & 54 & $\mathrm{~F}$ & AMI & ОРСАВ & 14 & 1 \\
\hline 5 & 75 & $\mathrm{~F}$ & $A D$ & $\begin{array}{c}\text { Graft interposition } \\
\text { of aorta }\end{array}$ & 21 & 1 \\
\hline 6 & 51 & M & $\begin{array}{l}\text { Mitral valve } \\
\text { regurgitation }\end{array}$ & $\begin{array}{l}\text { Mitral valve prosthesis, } \\
\text { maze operation }\end{array}$ & 6 & 0 \\
\hline
\end{tabular}




\section{Fig. 1. Case 4}

A 54-year-old man with sternal wound separation and mediastinitis. (A) Preoperative CT poststernal fluid collection (blue arrow) was noted when mediastinitis was diagnosed. (B) Intraoperative view of the sternal wound, which was debrided of foreign material and necrotic tissue. (C) Intraoperative view of the advanced pectoralis major muscle for coverage of the defect. (D) Two weeks after surgery.
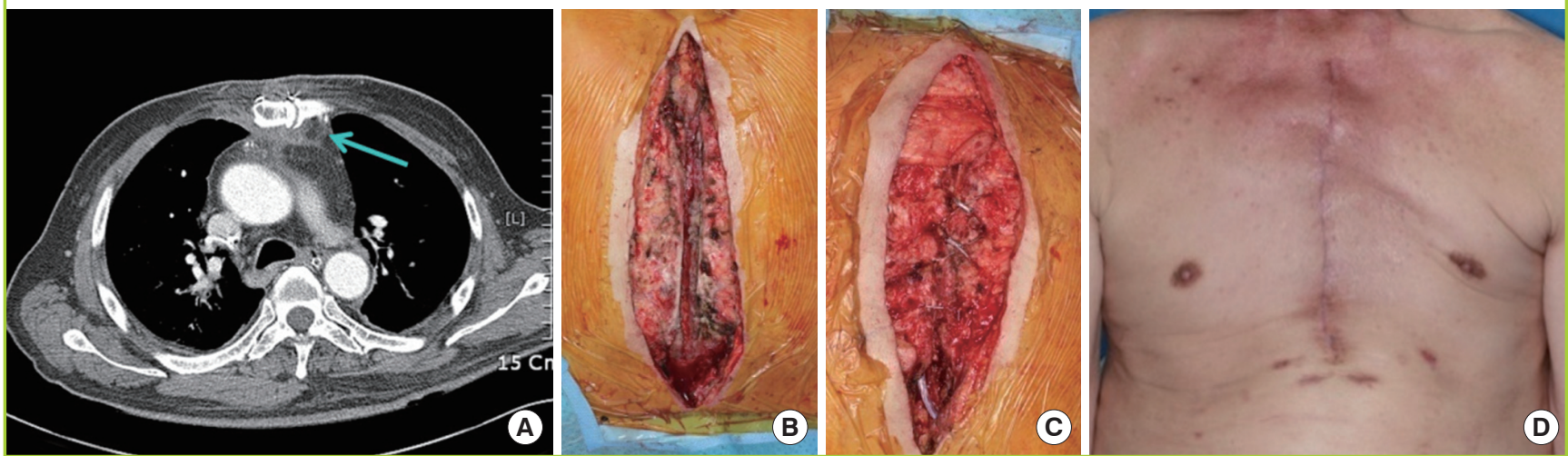

Fig. 2. Case 6

A 51-year-old man with poststernotomy mediastinitis. (A) Preoperative CT: poststernal fluid collection (blue arrow) was noted when mediastinitis was diagnosed. (B) Intraoperative view of the sternal wound, which was debrided of foreign material and necrotic tissue. (C) Intraoperative view of the advanced pectoralis major muscle for coverage of the defect. (D) Two weeks after surgery.
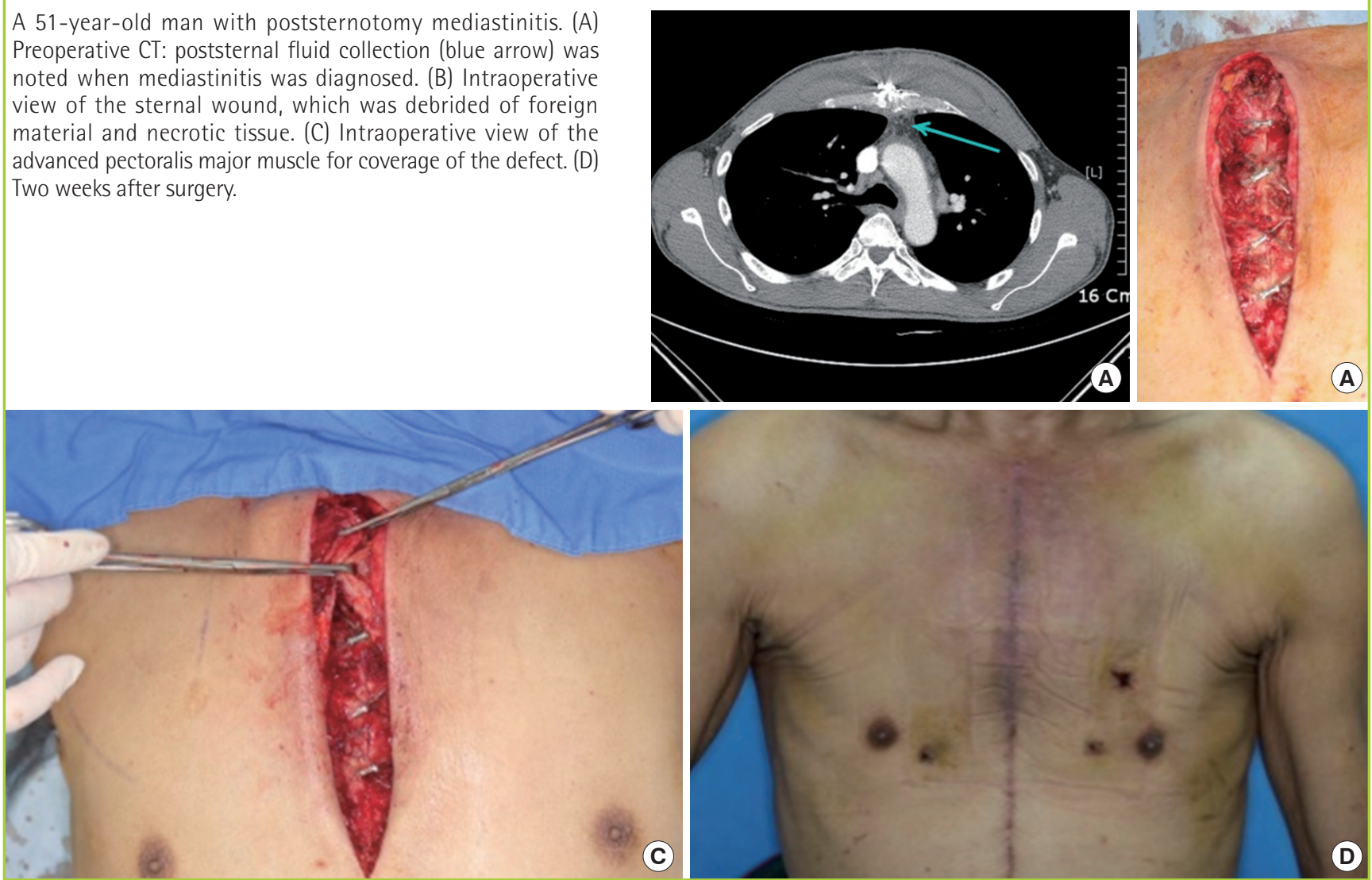

ing in age from 51 to 75 years (mean age, 61 years). Four patients underwent coronary artery bypass grafting, one patient had a graft interposition of the aorta, and one patient underwent surgery for mitral valve regurgitation. All wounds were located on the upper two-thirds of the sternum. The mean interval between diagnosis and debridement was 0.83 days (range, 0 to 2 days).

The procedure was successfully performed in 6 patients. The mean time of surgery was 3 hours. After immediate debridement and reconstruction with pectoralis major flaps, the inflammatory markers (ESR and CRP) (Table 2) were normalized on day 7 after operation, sternal stability was noted, and the poststernal fluid collections were found to be reduced on follow-up CT scans on post-surgical day 14. By postsurgical week 3 (range, 1-3 weeks), no growth of organisms was found in the wound cul- 


\section{Fig. 3. Case 1}

A 55-year-old man with anterior chest wall defect caused by mediastinitis after coronary artery bypass graft surgery. (A) Preoperative sternal wound with mediastinitis. (B) Preoperative CT: poststernal fluid collection (blue arrow) and sternal instability were noted when mediastinitis was diagnosed. (C) Intraoperative view of the bone defect after debridement. (D) Intraoperative view of the advanced pectoralis major flap designed for the bone gap.
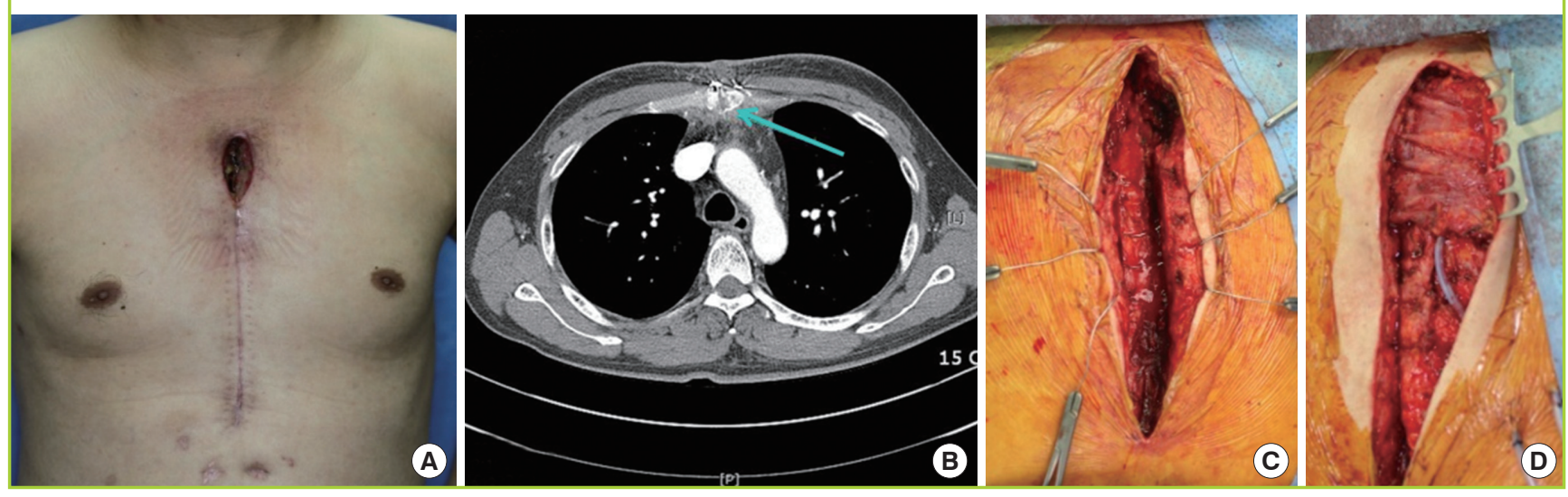

Table 2. Wound culture and inflammatory marker

\begin{tabular}{|lccccc|}
\hline & \multicolumn{2}{c}{ Wound culture } & & \multicolumn{2}{c}{ ESR/CRP } \\
\cline { 2 - 3 } \cline { 5 - 6 } Case & $\begin{array}{c}\text { Before } \\
\text { surgery }\end{array}$ & $\begin{array}{c}\text { After } \\
\text { surgery }\end{array}$ & & $\begin{array}{c}3 \text { days } \\
\text { before } \\
\text { surgery }\end{array}$ & $\begin{array}{c}7 \text { days } \\
\text { after } \\
\text { surgery }\end{array}$ \\
\hline 1 & MRCNS & No growth & & $46 / 8.26$ & $22 / 0.27$ \\
2 & MRCNS & No growth & & $33 / 16.32$ & $25 / 1.04$ \\
3 & MRCNS & No growth & & $57 / 21.47$ & $21 / 0.81$ \\
4 & MRCNS & No growth & & $38 / 8.17$ & $34 / 1.70$ \\
5 & $\begin{array}{c}\text { Enterobacter } \\
\text { cloacae }\end{array}$ & No growth & & $52 / 5.03$ & $31 / 0.75$ \\
6 & No growth & No growth & & $38 / 12.89$ & $15 / 1.14$ \\
\hline
\end{tabular}

ESR, erythrocyte sedimentation rate; CRP, C-reactive protein; MRCNS, methicillin resistant coagulase-negative staphylococci.

tures (Table 2). There were no major wound complications such as hematoma or complete wound dehiscence, but one patient required a skin graft for a $2 \times 1 \mathrm{~cm}$ superficial skin flap necrosis. No mortalities or recurrences occurred after the definitive surgery.

Although minimal weakening of the shoulders and bulging of the chest wall were noted in some patients, no patient complained of shoulder pain or a limitation of motion.

\section{DISCUSSION}

The treatment of sternal wound infections has evolved considerably over the past 40 years [5]. Because severe sternal wound complications can occur after sternotomies, the optimal management for sternal wound infections remains an important topic. The management for poststernotomy mediastinitis can be classified based on conservative care and the type of surgical technique.

Conservative care includes the use of antibiotics, frequent wet dressing changes, incisional drainage, and vacuum-assisted closure (VAC). The use of sub-atmospheric pressure in the wound environment for wound closure with VAC was developed in 1997 by Morykwas et al. [6]. The application of negative pressure increased the blood flow, increased the rate of granulation tissue formation, and decreased tissue bacterial counts $[1,6]$ VAC is actively and successfully used in many hospitals as the first-line therapy for poststernotomy mediastinitis. However, the depth of the sternal infection limits the role of the wound vacuum, as either the sole therapy for more superficial wounds or as adjuvant therapy for deeper wounds [1].

Most patients undergoing coronary artery bypass grafting have multiple medical problems, including diabetes, hypertension, steroid use, and pulmonary failure. Thus, the results achieved through conservative care have often been limited by these other medical conditions; in such cases, surgical procedures are needed for poststernotomy mediastinitis [5].

Since the 1980s, debridement and reconstruction with muscle or myocutaneous flaps has gained popularity in the treatment of poststernotomy mediastinitis $[7,8]$. Muscle flaps are well vascularised tissue and increase resistance against infection. Coverage with a muscle flap also obliterates dead space [8].

Currently, the most commonly used muscle for closing an infected sternal wound is the pectoralis major [9]. Pectoralis major muscle flaps require no additional incisions, produce symmetric results without functional problems or aesthetic deformities, and do not require intact internal mammary arteries. The pectoralis major muscle flap can be transposed into the mediastinum based on the thoracoacromial pedicle as an advancement flap or as a turnover flap based on the perforators of the internal mammary artery [5]. Some surgeons use the pectoralis 
major muscle as a turnover flap to fill and obliterate the dead space of the mediastinum [10]. This procedure requires dividing the humeral insertion of the muscle and folding this lateral portion of the muscle into the mediastinum. The procedure may result in a contour deformity and tension of the anterior chest wall. Furthermore, the morbidity increases with more complex procedures. In 1994, Ringelman et al. [11] reported postoperative complaints after pectoralis flap reconstruction of sternal wounds. Many of the reconstructions involved pectoralis muscle flap transposition or turnover. Ringelman et al. reported complaints of pain, shoulder weakness, and deformities in 25-50\% of patients over 4 years. It is also considered risky to use pectoralis major muscle flaps when the ipsilateral internal mammary artery has been used for coronary artery bypass grafts (CABGs) [5]. For these reasons, some plastic surgeons prefer to cover the sternal defect with pectoral flap advancement when there is no need for release of the humeral insertion of the pectoralis major muscle and the muscle and skin can be raised as one unit off the chest wall and advanced [5].

Rectus abdominis muscle flaps have been used for reconstruction of lower one-third sternal defects, which are difficult to manage with pectoralis major muscles $[8,12]$. However, the increased use of the internal mammary artery for CABGs and the complications associated with excised rectus abdominis muscles, such as abdominal hernias, implies that using a rectus abdominis muscle for reconstruction may not be a logical choice.

A combination pectoralis major and rectus abdominis bipedicled muscle flap is called a robust flap. It can completely cover large sternal defects. Moreover, the robust flap has a good blood supply from the thoracoacromial artery superiorly and the deep inferior epigastric artery inferiorly. If the ipsilateral internal mammary artery has been used for a CABG, then the blood supply to the flap will not be compromised [13]. However, this combined flap has disadvantages, including a long operative time and abdominal hernias.

There are other flap choices that are similar to the combined pectoralis major and rectus abdominis bipedicled muscle flap. The omental flap has a rich blood supply that can support a skin graft on the surface and revascularize the surrounding tissues, and requires a laparotomy, thus communicating a sterile cavity with a contaminated cavity. The conditions, such as potential adhesions, cannot be stated until the time of surgery. Furthermore, a previous abdominal surgery can preclude the use of an omental flap, and the right gastroepiploic artery, in which we prefer to base the flap, could have been used previously for myocardial revascularization [12].

The duration between diagnosis of poststernotomy mediastinitis and surgery as well as the choice of an appropriate flap are key to successful results. In 2009, Cabbabe and Cabbabe [14] analysed 583 patients with deep sternal wound infections, and compared two groups of patients after debridement and pectoralis muscle flap reconstruction. Of the 497 patients referred immediately, 22 (4.4\%) patients required mechanical ventilation for an average of 4 days, eight (1.6\%) required tracheotomy, 13 (2.6\%) developed stage III/IV pressure sores, 24 (4.8\%) developed major wound dehiscence, and none (0\%) required skin grafting; the average length of hospital stay was 4.7 days, and five (1\%) died. Of the 86 patients with a delayed referral, 40 (46.5\%) required mechanical ventilation for an average of 18.3 days, 31 (36\%) required tracheotomy, 20 (23.3\%) developed stage III/ IV pressure sores, 12 (14\%) developed major wound dehiscence, and $9(10.5 \%)$ required skin grafts; the average length of stay was 19.3 days, and 4 (4.7\%) died [14].

Like the preceding report, we also achieved better results from immediate debridement and reconstruction after diagnosis of poststernotomy mediastinitis. The mean interval between diagnosis and debridement was 0.83 days (range, 0 to 2 days), shorter than the interval in the preceding immediate group (within 4 days). Despite several limitations to our study, such as the short follow-up period, small sample size, and poor measures of muscle power, of the 6 patients, none (0\%) died and none (0\%) developed major complications. Because delayed debridement or coverage can lead to residual dead space, persistent or recurrent infections, especially nosocomial infections from a long hospital stay and intensive care unit care can occur when the ideal time for wound management has been missed. Thus, in cases of deep sternal wound infections involving the upper two-thirds of the sternum, immediate debridement and reconstruction after diagnosis with pectoralis major muscle flaps should be the treatment of choice to reduce inflammation, morbidity, and mortality.

\section{REFERENCES}

1. Domkowski PW, Smith ML, Gonyon DL Jr, et al. Evaluation of vacuum-assisted closure in the treatment of poststernotomy mediastinitis. J Thorac Cardiovasc Surg 2003;126:386-90.

2. Mangram AJ, Horan TC, Pearson ML, et al. Guideline for prevention of surgical site infection, 1999. Hospital Infection Control Practices Advisory Committee. Infect Control Hosp Epidemiol 1999;20:250-78.

3. Pasaoglu I, Arsan S, Yorgancioglu AC, et al. A simple management of mediastinitis. Int Surg 1995;80:239-41.

4. Lopez-Monjardin $\mathrm{H}$, de-la-Pena-Salcedo A, Mendoza-Munoz $\mathrm{M}$, et al. Omentum flap versus pectoralis major flap in the treatment of mediastinitis. Plast Reconstr Surg 1998;101: 1481-5. 
5. Jurkiewicz MJ, Bostwick J 3rd, Hester TR, et al. Infected median sternotomy wound: successful treatment by muscle flaps. Ann Surg 1980;191:738-44.

6. Morykwas MJ, Argenta LC, Shelton-Brown EI, et al. Vacuum-assisted closure: a new method for wound control and treatment: animal studies and basic foundation. Ann Plast Surg 1997;38:553-62.

7. Pearl SN, Weiner MA, Dibbell DG. Sternal infection after cardiac transplantation: successful salvage utilizing a variety of techniques. J Thorac Cardiovasc Surg 1982;83:632-4.

8. Yoo YC, Chung SI, Yang WY, et al. Treatment of acute sternomediastinitis using regional muscleflaps. J Korean Soc Plast Reconstr Surg 2003;30:345-50.

9. Ascherman JA, Patel SM, Malhotra SM, et al. Management of sternal wounds with bilateral pectoralis major myocutaneous advancement flaps in 114 consecutively treated patients: refinements in technique and outcomes analysis. Plast Reconstr Surg 2004;114:676-83.
10. Nahai F, Morales L Jr, Bone DK, et al. Pectoralis major muscle turnover flaps for closure of the infected sternotomy wound with preservation of form and function. Plast Reconstr Surg 1982;70:471-4.

11. Ringelman PR, Vander Kolk CA, Cameron D, et al. Longterm results of flap reconstruction in median sternotomy wound infections. Plast Reconstr Surg 1994;93:1208-14.

12. Mathes SJ, Eshima I. The principles of muscle and musculocutaneous flaps. In: McCarthy JG, editor. Plastic surgery. 3rd ed. Philadelphia: W. B. Saunders; 1990. p. 379-411.

13. Greig AV, Geh JL, Khanduja V, et al. Choice of flap for the management of deep sternal wound infection: an anatomical anatomical classification. J Plast Reconstr Aesthet Surg 2007;60:372-8.

14. Cabbabe EB, Cabbabe SW. Immediate versus delayed onestage sternal debridement and pectoralis muscle flap reconstruction of deep sternal wound infections. Plast Reconstr Surg 2009; 123:1490-4. 